\title{
Opinión De La Práctica Docente De Estudiantes En Formación Inicial En Educación Especial
}

\section{Opinion Of The Teaching Practice Of Students In Initial Training In Special}

\author{
Escobar Rodas Alma Rosa1, Espinoza Ochoa Nayeisy²
}

\begin{abstract}
1- Licenciatura en Educación Especial. Docentes-investigadores Realiza diagnóstico respecto a la "Opinión de la práctica docente de estudiantes en formación inicial en educación especial" de $2^{\circ}$ sem. Escuela normal Fray Matías de Córdova. Tapachula, Chiapas" México.

2 - Licenciatura en Educación Especial. Realiza diagnóstico respecto a la "Opinión de la práctica docente de estudiantes en formación inicial en educación especial” de $2^{\circ}$ sem. Escuela normal Fray Matías de Córdova. Tapachula, Chiapas” México.
\end{abstract}

\section{Resumen}

La formación profesional que llevan los estudiantes que se forman como Licenciados en Educación Especial consta de cuatro años de formación. Se divide en ocho semestres: seis de formación académica y el último año que es séptimo y octavo semestre, se enfocan en el trabajo docente, donde los alumnos y alumnas se preparan con su documento recepcional. En el cuarto semestre se escoge área de formación específica. Esta escuela normal oferta las áreas de formación: intelectual, auditiva y de lenguaje. En los primeros seis semestres de la licenciatura los estudiantes por cada semestre cursan ocho materias en clases áulicas, los días sábados llevan taller de braille y lengua de señas mexicanas, junto con otras promotorías, ya que se tienen que cumplir con seis promotorías antes de que termine el sexto semestre.

Palabras clave: práctica docente, estudiantes en formación, educación especial.

\begin{abstract}
Abstact
Vocational training leading students formed as graduates in special education consists of four years of training. It is divided into eight semesters: six of academic training and the last year's seventh and eighth semester focus on teaching work, where students are preparing their reception document. In the fourth semester specific training area is chosen. This normal school offering training areas: intellectual, hearing and language. In the first six semesters of undergraduate students for each semester enrolled in eight classes in courtly classes on Saturdays lead workshop braille and Mexican Sign Language, along with other Promontories as they have to meet six Promontories before end of the sixth semester.
\end{abstract}

Keywords: teaching practice, students in training, special education 


\section{Contextualización}

En base en nuestro proceso académico mexicano en la licenciatura de educación especial, podemos decir que se apoya de varios fundamentos teóricos de diversas ramas como: la pedagogía, la psicología, la medicina, la sociología, la fisioterapia, entre otras ramas que en cierto momento pueden colaborar con las necesidades educativas que se presenta en el alumno, con o sin discapacidad. De las cuales derivan autores importantes como: Jean Piaget, con sus diferentes teorías y aportaciones a la educación y al desarrollo y proceso cognitivo de los niños. Lev Vigotsky, con sus teorías de aprendizaje; así como muchos autores que se encuentran dentro del campo formativo de la educación especial.

En el proceso de reconocer cómo se ha estudiado la formación inicial, revisamos algunas investigaciones que han abordado el tema, las cuales nos fortalecieron para precisar el objeto de estudio. A partir de la revisión que hicimos de las investigaciones se encontraron diferencias en la manera en que abordan la temática, tanto desde el enfoque como de la metodología.

Barrera (2007) plantea una investigación descriptiva sobre la formación de docentes que cursan el sexto año, donde se indagó a docentes y directivos, de 12 instituciones que radican en
Bolivia. Plantea la autora que la formación docente inicial funciona a través de conocimientos pedagógicos con la investigación acción. También habla de los nuevos establecimientos que trae consigo la reforma educativa acerca de las competencias generales para la formación del docente: estimular la búsqueda de información y reflexión en los alumnos; influir en el proceso educativo y qué hacer para superarlos; el desempeño académico y algunas de las características que desarrolla un docente para emitir a las nuevas generaciones (Barrera Andaur S. , 2007).

Pérez Mendoza realiza una investigación sobre los procesos para la obtención de los distintos panoramas que tienen los practicantes. Enfocándose a las vivencias de estudiantes de la licenciatura de geografía en la Escuela Normal Superior de México, durante su formación docente. Para obtener la información consultó a profesores titulares de grupo, practicantes, asesores. Los resultados que presenta la autora, nos da a conocer las actitudes que adquieren los estudiantes durante las prácticas, al igual que las funciones que cumplen.

Pérez R. (2010) nos habla sobre el proceso de prácticas de un docente y el acercamiento con la realidad para analizar el proceso de enseñanzaaprendizaje; la importancia de conocer los programas de la educación básica, y el plan que 
corresponde a dicha licenciatura. Para realizar la investigación utilizó un método de revisión teórica para obtener reflexiones sobre las prácticas del docente.

Inzunza H. (2011) investigó sobre la formación docente inicial y en servicio, y las transformaciones que han tenido, así como la forma en que las políticas educativas trataban de evitar la unión de maestros y manifestaciones; también menciona sobre las condiciones con las que un docente debe contar para impartir aprendizaje y conocimientos a los educandos.

Con base a las revisiones de las investigaciones que abordan la formación docente, situamos el contenido del trabajo que se presenta a la Perspectiva de la práctica docente que tienen los estudiantes de la licenciatura en educación especial, de la escuela normal Fray Matías de Córdova, Tapachula. Dado que los estudiantes de la licenciatura en educación especial, de la escuela Normal Fray Matías de Córdova, realizan prácticas docentes de observación y ayudantía dos veces por semestre.

Respecto al planteamiento anterior, se ha formulado la siguiente pregunta ¿Cuál es la opinión de la práctica docente que realizan los estudiantes de la licenciatura en educación especial, de la escuela normal Fray Matías de Córdova, Tapachula, Chiapas?
Objetivo general: Conocer la experiencia que han tenido los dicentes de distintos grados durante su estancia en la carrera de Educación Especial y a su vez basándonos en fundamentos teóricos.

Objetivos Específicos: Indagar cuales son los planteamientos teóricos sobre la educación especial; analizar la perspectiva de la práctica docente. Reconocer la opinión de los estudiantes de la licenciatura en Educación Especial respecto a la práctica docente que realizan.

De acuerdo a metodología de la Investigación este concepto se define como "conjunto de estrategias y tácticas que se siguen en una investigación y que incluye el método, las técnicas, los instrumentos y las herramientas" (Perroni Castellanos \& Gúzman Piedra, 2013. Pag. 59), dicha secuencia se ha desarrollado en esta investigación. En este caso la investigación es cualitativa, como dice Fernández Pita "los investigadores hacen registros narrativos de los fenómenos que son estudiados mediante técnicas participante y las entrevistas no estructuradas" (Fernández, S, 2002, pag. 1), que durante este proceso fueron realizados.

Para la realización de la investigación, se usó de la técnica del cuestionario cualitativo, para ello solo participaron 20 estudiantes, de un total de 104 alumnos que cursan la Licenciatura en Educación Especial. Participaron cinco estudiantes por semestre. La manera en que se seleccionaron solo se consideró el criterio de 
participación voluntaria de cinco estudiantes por semestre.

Una vez obtenido los estudiantes voluntarios se les aplicó un cuestionario que contenía cuatro preguntas enfocadas en las prácticas docentes de observación y ayudantía que se realizan en el semestre. Las opiniones que proporcionaron han sido analizadas.

Estas opiniones las encontraremos más adelante en el epígrafe Análisis de la opinión respecto a las prácticas de observación y ayudantía en la licenciatura en Educación Especial, en donde hemos asignado una clave a cada respuesta del estudiante, la clave es D de docente y uno de los siguientes números $(2,4,6,8)$ los cuales corresponden al semestre que actualmente cursan.

Hipótesis: La opinión que tienen de la práctica docente que realizan los estudiantes de la licenciatura en educación especial, de la escuela normal Fray Matías de Córdova, Tapachula, Chiapas. Es que, esta es trasmisora de conocimientos, en las cuales analizan las diferentes teorías y opiniones de autores de la enseñanza.

\section{Marco teorico: Reconocer el} planteamiento teórico respecto a las Necesidades Educativas Especiales, amplío nuestros horizontes respecto al diagnóstico que presentamos sobre las prácticas docentes en la formación docente inicial en educación especial.

\section{Qué Son Las Nee, Teoría y Opinión Del Discente.}

Con base al planteamiento teórico de la materia Introducción a la Educación Especial, el término Necesidades Educativas Especiales (NEE) supera la concepción clásica de la educación especial atendida como la educación de los alumnos con deficiencias físicas, psíquicas o sensoriales y, por tanto, exclusiva de unos pocos, para avanzar hacia un nuevo concepto en el que se contemplan (además de otras circunstancias) la respuesta que incluye también a los alumnos que presentan dificultades en la adquisición de los aprendizajes debido a problemas de tipo madurativo, deprivación social y cultural (Secretaría de Educación Pública, 2005).

A partir de la revisión que se ha hecho de las teorías que se proponen en la asignatura se plantean las siguientes definiciones de las NEE.
Según Brennan (1968) señala que hay una
necesidad educativa especial cuando una
deficiencia (física, sensorial, intelectual,
emocional, social o cualquier combinación de
estas) afecta al aprendizaje hasta tal punto que
son necesarios algunos o todos los accesos especiales
al curriculum, a un curriculum especial o
modificado, o bien una adecuación de las
condiciones de aprendizaje. La necesidad puede
presentarse desde leve a aguda y puede ser

34 ROSA, E.R.A., Espinoza Ochoa NAYEISY, E.O.: De La Práctica Docente De Estudiantes En Formación Inicial En Educación Especial. 
permanente o presentarse temporalmente en alguna fase del desarrollo.

Similar a lo que nos menciona Hegarty indica que el grupo de sujetos considerados de necesidades educativas especiales "incluye a quienes tienen impedimentos físicos o sensoriales, a aquellos que comparados con los pares de su edad tienen dificultades en su aprendizaje o al comunicarse, como también a aquellos cuya conducta no puede ser aceptada sin problemas en las aulas o en escuelas regulares. Lo que estos niños tienen en común es una mayor o menor necesidad de que se les brinde una oferta educativa especial que esté por encima de la que, la generalidad de las escuelas, ofrecen a la mayoría de los estudiantes".

\section{El termino necesidades educativas} especiales ha ido impregnando diversos marcos legislativos y significando nuevas formas de entender la educación especial. Es un término amplio, aunque no exacto de críticas, se fundamenta en el concepto de necesidades, entendiendo ésta como un continuo (Sánchez Palomino, 2000), y que podríamos definir a partir del análisis de tres perspectivas diferentes y complementarias, esto es aludiendo al curriculum, al concepto de necesidades como un continuo anti etiquetador y a la etiología (Secretaría de Educación Pública, 2005).

Según Sánchez Palomino y Torres Gonzáles la educación especial consiste en: un alumno tiene necesidades educativas especiales cuando presenta dificultades mayores que el resto de los alumnos para acceder a los aprendizajes que se determinan en el curriculum que le corresponde por su edad (bien por causas internas, por dificultades o carencias en el entorno socialfamiliar o por una historia de aprendizaje desajustada) y necesita, para compensar dichas dificultades, adaptaciones de acceso y/o adaptaciones curriculares significativas en varias áreas de ese curriculum".

Decir que un alumno o una alumna presenta Necesidades Educativas Especiales es una forma de decir simplemente que, para el logro de los fines de la educación, no son suficientes las actuaciones habituales que su profesor o profesora desarrolla con la mayoría de los alumnos del grupo y que, por ello, tiene que revisar su acción educativa y adecuarla a las necesidades particulares el alumno o la alumna en cuestión" (MEC,1994:22).

Las necesidades educativas especiales tienen su origen en causas relacionadas fundamentalmente, con el contexto social o cultural, con la historia educativa y escolar de los alumnos o con condiciones personales asociadas, bien a una sobredotación en cuanto a capacidades intelectuales, bien a una discapacidad psíquica, sensorial o motora o trastorno grave de conducta" (BOE,2-6-1995). (Secretaría de Educación Pública, 2005, pp. 137-138). 
Con base a lo que dicen los autores, un alumno presenta una Necesidad Educativa Especial (NEE) cuando su proceso de desarrollo es diferente al de sus pares y por lo tanto es necesario hacerle adaptaciones al curriculum de acuerdo a su edad y a la NEE que presenta el niño o niña, para que su progreso educativo sea favorable en cuanto a la adquisición de conocimientos.

García Pastor (1995:43) precisa los dos rasgos esenciales de este concepto cuando indica que "es necesario que el concepto de necesidades educativas especiales se redefina incluyendo dos dimensiones esenciales: la dimensión interactiva (la necesidad se define en relación con el contexto donde se produce) y la dimensión de relatividad (la necesidad hace referencia a un espacio determinado y a un tiempo determinado, no es universal ni permanente)" (Secretaría de Educación Pública, 2005, p.138).

Con base al planteamiento teórico que hay sobre las necesidades educativas especiales, el plan de estudios 2004 de la licenciatura en Educación Especial en México considera lo siguiente para la formación del docente en esta área.

El área está formada por 36 cursos, de duración semestral, distribuidos a lo largo de los primeros seis semestres. La intensidad del trabajo semanal de cada asignatura varía de cuatro a seis horas semanales repartidas en varias sesiones. Las actividades planteadas por los cursos se realizan en la escuela normal.

Actividades de Acercamiento a la Práctica Escolar. Está formada por seis cursos, que se desarrollan del primero al sexto semestres, con una intensidad de seis horas semanales cada uno. Mediante la observación y la práctica docente, y con la orientación de los maestros de las escuelas normales, estas actividades asocian el aprendizaje logrado en las distintas asignaturas con el conocimiento de los servicios de educación especial y de las escuelas de educación básica regular.

La realización de las jornadas o estancias de práctica docente se ejecutan en los servicios escolarizados de educación especial y en los planteles de preescolar, primaria o secundaria que cuenten con el servicio de educación especial, con la preparación de las mismas y el análisis de las experiencias obtenidas, actividades que se llevan a cabo en la escuela normal.

Actividades de Práctica Intensiva en Condiciones Reales de Trabajo. El área comprende dos periodos de trabajo docente en un servicio de educación especial, los cuales se realizan en los últimos dos semestres de la licenciatura. En estos periodos los estudiantes serán corresponsables de la atención de los alumnos que presenten necesidades educativas especiales - preferentemente con la discapacidad 
correspondiente al área de atención que cursanen uno o más grupos de un servicio escolarizado de educación especial o de un servicio de apoyo a un plantel, en alguno de los niveles de educación básica que cuenten con niños y adolescentes integrados. En el desarrollo de esta actividad contarán con la tutoría continua de el o los profesores de apoyo o de los titulares de los grupos -según sea el caso-, responsables de educación especial.

Una vez aclarado la teoría que fundamenta las necesidades educativas especiales y las prácticas docentes que se realizan en el área, en el siguiente apartado se plantea el análisis de los cuestionarios que se aplicaron a los estudiantes de la licenciatura en Educación Especial.

\section{2 - Análisis De La Opinión Respecto A Las Prácticas De Observación Y Ayudantía En La Licenciatura En Educación Especial.}

Para logar la intervención educativa se requiere de la participación de los docentes y en ello es en lo que nos enfocaremos, actualmente hay una gran demanda de maestros, pero no tanto en lo que confiere a educación especial. Por ende hay estuantes en formación y de acuerdo a lo que nos mencionan los que participaron en este diagnóstico ellos han ingresado a esta licenciatura por qué:
D6: "En el mundo hay personal (niños), que requieren apoyo específico, pero en la actualidad no hay tantos docentes capacitados para atenderlos; por ello elegí la carrera, para prepararme y apoyar a las personas que sufren rechazo por la sociedad".

D8: "Seré muy sincero, no tenía otra opción, mi familia no tenía los recursos para cumplir mis deseos, pero actualmente estoy enamorado y agradecido con Dios por estar en este espacio y vivir las experiencias que hasta ahora me mantienen con muchas ganas de continuar".

D4: "Quiero ser una maestra que pueda ayudar a los alumnos a trabajar con la integración e inclusión y salir de lo rutinario con respecto a clases; lo que quiero es que ellos aprendan y yo aprender de ellos. Y yo sé que lo voy a lograr".

De acuerdo con lo que menciona el estudiante "D8" sobre el rechazo, es porque nosotros mismos hemos inculcado esta costumbre, si desde un inicio se nos fomentara la ideología de que somos completamente iguales no habría por qué preocuparnos de si existen diferencias, nos veríamos unos a otros completamente iguales sin hacer distinción alguna.

Reflexionamos a partir de lo que comentan los docentes que, al inicio del estudio de esta carrera, la tenían contemplada como una segunda opción o porque creen que con atender a niños "especiales" realizan una obra caritativa sin embargo como menciona la antología de Propósitos y Contenidos de la Educación Básica educar es más que enseñar a leer, a escribir o a calcular. Es preparar al individuo para el mundo, 
para que él pueda verlo, juzgarlo y transformarlo" (Secretaría de Educación Pública, 2005, p.115).

Respecto a las teorías que se revisan dentro del aula de clases y a la realización de las prácticas de observación y ayudantía se obtuvieron las siguientes opiniones:

D8: "La práctica es una oportunidad que tenemos durante nuestra formación inicial de vivir la experiencia de lo que aprendemos y estudiamos en la escuela normal, lleva implícito una variedad de aspectos entre teoría y práctica, la teoría sirve en ocasiones como un referente de las coincidencias con la realidad o en circunstancias específicas; es por ello que, no podemos generalizar mediante la afirmación que toda la teoría se llevara a cabo al pie de la letra. En numerosas ocasiones la teoría es lo ideal de aquello que se quiere ver reflejado a vista de la sociedad; sin embargo, ante la carencia de ciertas condiciones esenciales para su cumplimiento, la relación entre teoría-practica se enmarca en los planos convergente o divergente. Resumido el asunto: la sociedad siempre buscara lo ideal (debe ser-teoría), para ello, debe partir de la realidad (serpractica) que se vive en cada contexto según las necesidades presentes".

D8: "Que lamentablemente las teorías manejadas en clase son resultados de estudios realizados en unas situaciones muy diferentes a la de nuestro país en cuanto a calidad educativa y atención que se brinda, esto es resultado de la mala administración que brinda el gobierno. Un gobierno que maneja teóricamente una educación basada en competencias. Una educación copia directa de países considerados como los más desarrollados del mundo en el que la educación es muy importante para lograr el éxito. Los grupos de clase no son tan numerosos como en México y existen diversos especialistas que lo atienden. Deberíamos estudiar teorías apegadas a nuestra realidad, a nuestra pobreza, a nuestras necesidades y no crearnos ideas erróneas sobre la educación. No es conformismo, es la actualidad del país. Una educación de calidad requiere un gobierno de calidad. ¡Claro que se puede!

En base a lo que mencionan los docentes, coinciden en que la práctica va más allá de las teorías y sus altas expectativas que desean cumplir en el proceso educativo, ambos términos son muy diferentes, puesto que las experiencias que se viven no se asemejan a los conocimientos de las teorías analizadas en el proceso de formación docente, o al menos no se perciben en México.

\section{3 - Inicio De Prácticas}

Después de un periodo en el que se revisa la teoría se lleva a cabo las prácticas docentes y es de nuestra intención dar a conocer con que finalidad se realizan: 
D8: Con diversas finalidades que a continuación se enlistan:

1. Asimilar o contrastar los referentes teóricos con la realidad educativa.

2. Presenciar lo que ocurre durante la jornada escolar.

3. Interactuar con los protagonistas del proceso escolar (director, maestros, alumnos, padres/madres de familia, comunidad etc.)

4. Conocer diferentes contextos en que se brinda atención educativa a alumnos que presentan Necesidades Educativas Especiales (NEE), con o sin discapacidad.

5. Adquirir conocimientos, habilidades y actitudes para desempeñar su profesión docente en un futuro inmediato.

6. Poner en práctica los saberes adquiridos durante mi estancia en la escuela normal.

D6: Para poder llevar a la praxis todas aquellas teorías que hasta el momento se han revisado dentro la escuela normal. En consiguiente adquirir experiencias de lo que sucede en la jornada laboral; tiempos, actividades, personal, etc.

Este es un proceso por cual los estudiantes tienen que caminar para poner en marcha los procesos de intervención y no quedarse únicamente con las teorías las cuales sirven para poder comprender ciertos sucesos, acercándose a la realidad de las necesidades educativas especiales en la educación básica.

Al docente en formación, le es de gran importancia para su aprendizaje asistir a las prácticas de observación y ayudantía, porque aprenden cosas nuevas de los maestros y maestras que ya ejercen en la docencia, como:

D2: La forma en la que conciben la educación, me ha ayudado a entender como aprenden los niños, sobre todo aquellos que presentan Necesidades Educativas Especiales (NEE).

D4: Los métodos, estrategias y formas de trabajo. Como también la planeación que está llevando a cabo, para realizar sus estrategias actualizadas.

D4: Las diferentes formas, métodos $y$ estrategias que implementan dentro del aula de clases, y cómo impacta en el actuar o actitud de los alumnos hacia el proceso de enseñanza-aprendizaje.

D6: Una perspectiva de lo que es dedicar la vida a quien nos necesita; ver ese compromiso y vocación que pocos tienen como la motivación, para luchar por esos pequeños grandes logros.

D8: En lo pedagógico: estrategias didácticas, formas de evaluar, como relacionarse con los alumnos, capacidad de observación e interpretación de cosas 
profesionalmente; ética profesional y conocimientos generales.

D8: He aprendido, según los estereotipos que predominan en la sociedad, en este caso de la profesión docente, lo que se tiene que hacer o no para brindar una atención pertinente a las necesidades de los alumnos.

D8: Principalmente: lo que debo hacer, lo que no, el cuándo, el dónde, el cómo, el por qué y para qué hacerlo, y en muy pocas ocasiones, pero significativas el con qué hacerlo.

En base a las opiniones de los compañeros(as) de la Licenciatura en Educación Especial, podemos analizar que las prácticas que se realizan como parte del proceso académico, son de gran ayuda, tanto personal como profesional, ya que cada sujeto aprende y adquiere nuevas experiencias de los docentes y alumnos con los que interactúan y ponen en uso todo lo aprendido durante las clases. Dichos aprendizajes adquiridos por los maestros(as) son significativos para cada docente en formación, aun que de cada persona queda si tomarlos, hacerle adecuaciones o implementar los que el considere importantes en el proceso de enseñanza-aprendizaje con sus alumnos.

El maestro, nunca deja de aprender de sus semejantes durante el trayecto de su vida profesional, académica y personal, porque una cosa es lo que nos dicen los diferentes autores por medio de los libros pedagógicos, filosóficos, sociológicos, enciclopedias, entre otros más, y otra cosa es la que vive cada docente frente a su campo de trabajo, ya que de ahí es donde se adquieren esas experiencias y estrategias con las que imparten educación y atienden las necesidades de cada alumno, las cuales le proporcionan a cada docente en formación académica.

\section{4 - Conclusión}

La licenciatura para los estudiantes es de suma importancia desde el primer contacto, por ello desde que esta se cultiva se tiene que cuidar regándola todos los días con nuevos conocimientos y dándole cuidados con la ejecución de la práctica, la realidad es esa una planta no vive solo de agua es decir la teoría, también se tiene que explorar con la realidad, para que de ello se obtenga una buena cosecha con los resultados obtenidos de tu propio trabajo.

Esta investigación nos permite conocer lo que podemos encontrar o enfrentar en los siguientes semestres que nos hacen falta por recorrer, además de que podemos hacer una comparación de nuestros propios logros con los logros de los estudiantes que ya pasaron por esta misma etapa en la que nosotros nos encontramos

40 ROSA, E.R.A., Espinoza Ochoa NAYEISY, E.O.: De La Práctica Docente De Estudiantes En Formación Inicial En Educación Especial. 
actualmente y ver de qué manera podemos mejorar o si estamos transitando por un buen camino.

También lo consideramos como un ejercicio previo al momento en que tengamos que realizar nuestro documento recepcional, y para la ejecución de nuestros siguientes ensayos de las prácticas que continúan en los semestres restantes.

\section{Bibliografía}

Barocio, R. (2005). DISCIPLINA CON AMOR Cómo poner limites sin ahogarse en la culpa. México: PAX MÉXICO.

Barrera Andaur, S. (2007). EL DESARROLLO DE COMPETENCIAS Y LOS FACTORES INNOVADORES INTEGRADOS EN LA. REICE, 12.

Barocio, R. (2005). DISCIPLINA CON AMOR Cómo poner limites sin ahogarse en la culpa. México: PAX MÉXICO.

Barrera Andaur, S. (2007). EL DESARROLLO DE COMPETENCIAS Y LOS FACTORES INNOVADORES INTEGRADOS EN LA. REICE, 12.

Barrera Andaur, S. (2007). El desarrollo de competencias y los factores innovadores integrados en la formación docente inicial: una experiencia en institutos normales superiores de Bolivia. Redalyc, 12.

Definición.DE. (15 de marzo de 2016). http://definicion.de/integracion/.
Fernández, S, P. (2002). Investigación cuantitativa y cualitativa . Unidad de Epidemiología Clínica y Bioestadística. Complexo HospitalarioUniversitario Juan Canalejo. , 4.

H., I., Assaél B., J., \& Scherping V., J. (2011). FORMACIÓN DOCENTE INICIAL Y EN SERVICIO EN CHILE. Tensiones de un modelo neoliberal. Revista Mexicana de Investigación Educativa, 27.

PÉREZ MENDOZA, B. E. (s.f.). SABERES DE LOS ESTUDIANTES NORMALISTAS. Sujetos de la educación, 11.

Pérez R., S. M. (2010). REFLEXIONES SOBRE LAS PRÁCTICAS DOCENTES. SABER, 6 .

Perroni Castellanos, M. D., \& Gúzman Piedra, A. S. (2013). Metodología de la investigación. México, D.F.: Nueva imagen.

Secretaría de Educación Pública. (2004). Plan de estudios 2004. México: Consejo Nacional de Libros de Texto Gratuito.

Secretaría de Educación Pública. (2005). Desarrollo infantil y de los adolescentes. Aspectos generales. México: Consejo Nacional de Libros de Texto Gratuito.

Secretaría de Educación Pública. (2005). Introducción a la educación especial. México: Consejo Nacional de Libros de Texto Gratuito.

Secretaría de Educación Pública. (2005). Propósitos y Contenidos de la Educacion Básica I. México: Consejo Nacional de Libros de Texto Gratuito.

Vásquez Bronfman, A., \& Martínez, I. (1996). La socialización en la escuela. Una perspectiva etográfica. Barcelona: Paidós (Papeles de pedagogía, 26). 